\title{
Florística de um trecho de floresta de restinga no município de Guarapari, Espírito Santo, Brasil
}

\author{
André Moreira de Assis ${ }^{1,4}$, Luciana Dias Thomaz ${ }^{2}$ e Oberdan José Pereira ${ }^{3}$
}

Recebido em 12/11/2002. Aceito em 04/08/2003

\begin{abstract}
RESUMO - (Florística de um trecho de floresta de restinga no município de Guarapari, Espírito Santo, Brasil). O Parque Estadual Paulo César Vinha (PEPCV), em Setiba, município de Guarapari (ES), apesar de ser uma unidade de conservação em restinga com vários trabalhos desenvolvidos, apresenta lacunas quanto à composição florística de algumas comunidades vegetais. Durante o período de 1997 a 2000 foram realizadas coletas por meio de caminhadas em um trecho de floresta sobre os primeiros cordões no PEPCV, englobando cerca de quatro hectares, sendo incluídos espécimes de diferentes hábitos. Foram inventariadas 172 espécies pertencentes a 54 famílias, sendo Myrtaceae (25), Bromeliaceae (14), Orchidaceae (13), Sapotaceae (10), Lauraceae (7) e Rubiaceae (7) as de maior riqueza. O padrão de distribuição geográfica mais freqüente, segundo dados de literatura, foi o de espécies da costa Atlântica, seguido por aquelas com ampla distribuição e com ocorrência na costa atlântica e centro do Brasil, além das disjuntas e das endêmicas ao Estado do Espírito Santo. A similaridade florística entre a floresta estudada e outros trechos litorâneos demonstra que os índices estão relacionados com a distância geográfica entre as áreas. A presença de espécie endêmica ao Parque e outras ameaçadas de extinção reforça seu caráter conservacionista, devendo servir como incentivo para a criação de outras unidades de conservação nas restingas do Estado do Espírito Santo.
\end{abstract}

Palavras-chaves: florística, restinga, Espírito Santo, Brasil

\begin{abstract}
Floristic survey of a restinga forest in Guarapari, Espirito Santo State, Brazil). The Paulo César Vinha State Park (PCVSP), in Setiba, Guarapari Municipality (ES), is the best studied of all restinga conservation units in the state of Espírito Santo. But there is still much to be learned concerning the flora of some of the plant communities found here. From 1997 to 2000, plants were collected on random walks through approximately four hectares of forest that covers the outer beach ridges of the PCVSP. A total of 172 species were surveyed, belonging to 54 families. The most species-rich families are Myrtaceae (25 spp.), Bromeliaceae (14), Orchidaceae (13), Sapotaceae (10), Lauraceae (7), and Rubiaceae (7). The most common geographic distribution pattern, based on the literature, was that of Atlantic coast species, followed by widespread species, species found on both the Atlantic coast and in central Brazil, disjunct species and those endemic to Espírito Santo. Floristic similarity with other coastal areas is directly related to the distance between these areas. The presence of endemic and threatened species in the neighborhood of the Park reinforces the need for effective conservation of this area and provides the incentive to create other conservation units in the restingas of Espírito Santo State.
\end{abstract}

Key words: floristic, restinga, Espirito Santo, Brazil

\section{Introdução}

Planícies costeiras arenosas e tipos vegetacionais associados encontrados ao longo da costa brasileira representam um ecossistema bastante diversificado em fisionomia, florística e estrutura. Embora protegidas por unidades de conservação, são ameaçadas principalmente pela especulação imobiliária e extração de areia (Maciel 1990).

As comunidades vegetais de restinga foram descritas pioneiramente por Ule (1901), identificando distintas formações em Cabo Frio (RJ). Outros pesquisadores, posteriormente, empenharam-se na descrição fisionômica e nos levantamentos florísticos das restingas em diferentes pontos do litoral brasileiro, como Bresolin (1979) em Santa Catarina, Araujo \& Henriques (1984) no litoral do Rio de Janeiro, e Waechter (1985) no Rio Grande o Sul. Trabalhos que enfocam a composição florística das restingas estão referidos nas publicações de Araujo (1992), Fabris \& César (1996), Pereira \& Araujo (2000), dentre outros.

No Estado do Espírito Santo algumas das primeiras menções sobre restingas são encontradas nos trabalhos de Ruschi (1950) e Azevedo (1962) sobre a fitogeografia do Estado. Posteriormente, Pereira (1990) identificou as comunidades vegetais da restinga de Setiba, dando

\footnotetext{
${ }^{1}$ Museu de Biologia Prof. Mello Leitão, Av. José Ruschi, 4, CEP 29650-000, Santa Teresa, ES, Brasil (pouteria@ terra.com.br)

2 Setor Botânica, Universidade Federal do Espirito Santo, Av. Fernando Ferrari s/n, Goiabeiras, 29060-900, Vitória, ES, Brasil

3 Ciências Biológicas, FAESA, Rod. Serafim Derenze, 33115, São Pedro, CEP 29048-450, Vitória, ES, Brasil

4 Autor para correspondência: pouteria@ terra.com.br
} 
início à frente de estudos em diferentes pontos do litoral capixaba, como demonstrado nas publicações de Pereira \& Gomes (1994), Fabris \& César (1996), Pereira \& Zambom (1998), Pereira et al. (1998), Martins et al. (1999), Pereira \& Assis (2000) e Pereira et al. (2000).

A restinga de Setiba, no município de Guarapari, foi considerada área prioritária para conservação da biodiversidade, contemplada na categoria de alta importância biológica (Ministério do Meio Ambiente 2000) e, mesmo sendo a região litorânea melhor estudada no Estado, algumas comunidades possuem poucos estudos relacionados à sua flora, sobretudo as formações florestais, contempladas apenas pelos estudos de Fabris (1995) e Fabris \& César (1996).

Este estudo objetiva ampliar o conhecimento florístico de uma formação florestal de restinga situada no Parque Estadual Paulo César Vinha (PEPCV), ao sul do Estado do Espírito Santo considerando seus diferentes estratos e estabelecendo padrões de distribuição geográfica para suas espécies.

\section{Material e métodos}

O PEPCV compreende aproximadamente 1.500ha na restinga de Setiba, município de Guarapari, ES, localizado entre as coordenadas $20^{\circ} 33^{\prime}-20^{\circ} 38^{\prime} \mathrm{S}$ e $40^{\circ} 23^{\prime}-40^{\circ} 26^{\prime} \mathrm{W}$. Criado em 1990 pelo Decreto n. 2.993/1990 como Parque Estadual de Setiba, passou a ser denominado Parque Estadual Paulo César Vinha com a Lei n. 4.903/1994. O clima da região, segundo classificação de Köppen, é do tipo Aw tropical, com verão quente e chuvoso e inverno seco; a temperatura média anual é de $23,3^{\circ} \mathrm{C}$, a precipitação média anual é de $1.307 \mathrm{~mm}$ e a umidade relativa média anual é de $80 \%$ (Fabris 1995).

A formação florestal analisada localiza-se no primeiro cordão arenoso, próximo à estrada da entrada do Parque, afastada cerca de $150 \mathrm{~m}$ do mar, tendo como limite leste a comunidade arbustiva pós-praia e à oeste, a aberta de Clusia, mais afastada do mar (Pereira 1990). No sentido norte-sul a floresta é praticamente contínua sendo, em alguns pontos, cortada por trilhas perpendiculares ao mar.

O levantamento florístico concentrou-se em uma área de aproximadamente 4ha da floresta, onde foram coletados ramos férteis de indivíduos das Magnoliophyta, durante o período de 1997 a 2000, estando depositados no Herbário VIES, da Universidade Federal do Espírito Santo. O material foi identificado por meio de literatura especializada, consulta aos Herbários VIES e CVRD, e envio a especialistas de algumas famílias. Utilizou-se o sistema de Cronquist (1981) para a classificação das famílias, sendo mantida Leguminosae.

A análise da distribuição geográfica das espécies inventariadas na floresta de restinga do PEPCV foi realizada com informações de literatura para as várias regiões do território nacional, sendo consideradas apenas as determinações ao nível específico. Os padrões de distribuição geográfica das espécies seguiram proposta de Mori et al. (1981), conforme sua ocorrência nos ecossistemas brasileiros: (a) costa atlântica (floresta ombrófila densa, floresta estacional semidecídual, floresta ombrófila mista e restingas); (b) costa atlântica e região central do Brasil (caatinga e cerrado); (c) disjunta entre a costa atlântica e a região amazônica; (d) ampla distribuição nestes ecossistemas.

Visando o conhecimento das relações florísticas entre a floresta de restinga do PEPCV e diferentes trechos do litoral brasileiro, foi realizada análise de similaridade, utilizando o Índice de Sørensen (MuellerDombois \& Ellenberg 1974), considerando apenas estudos fitossociológicos, na tentativa de minimizar problemas metodológicos, principalmente critério de inclusão e esforço amostral nos estudos considerados. Assim, foram utilizadas as espécies arbóreas (diâmetro à altura do peito $\geq 4,8 \mathrm{~cm}$ ) da floresta de restinga do PEPCV inventariadas por Assis (2001) em estudo fitossociológico na mesma área de estudo, no total de 81 espécies, já desconsideradas aquelas determinadas apenas ao nível genérico e as imprecisas (cf. ou aff.).

\section{Resultados e discussão}

A floresta analisada no PEPCV tem dossel contínuo com altura média de $8 \mathrm{~m}$, apresentando diferenças principalmente no sub-bosque que ora encontra-se dominado por bromélias, principalmente Pseudananas sagenarius e Bromelia antiacantha, ora por indivíduos herbáceo-arbustivos de Arecaceae, Piperaceae e Rubiaceae.

O epifitismo é relativamente comum nesta floresta, com destaque para as Araceae, Orchidaceae e Bromeliaceae, presentes em grande número de indivíduos e espécies, além de outras famílias menos representativas em relação a este parâmetro, como Cactaceae e Piperaceae. Outro tipo de hábito ocorrente na floresta de Setiba é o de hemiepifítico, que apresenta comportamento de "mata-pau", como Coussapoa microcarpa (Cecropiaceae) e Clusia hilariana (Clusiaceae). 
$\mathrm{Na}$ floresta de restinga do PEPCV foram inventariadas 172 espécies distribuídas em 54 famílias (Tab. 1), sendo as mais importantes quanto ao número de espécies Myrtaceae (25), Bromeliaceae (14), Orchidaceae (13), Sapotaceae (10), Lauraceae (7), Rubiaceae (7), Moraceae (5) e Sapindaceae (5). Vinte e quatro famílias (44\% do total) estão representadas na área de estudo por apenas uma espécie, fato também observado em outros estudos na costa brasileira (Silva et al. 1994; Trindade 1991; Bastos 1996; Sugiyama 1998; Pereira \& Assis 2000). Este levantamento florístico representa um incremento de 105 espécies para a flórula do Parque, considerando os trabalhos de Pereira (1990), Pereira \& Araujo (1995), Fabris \& César (1996) e Fabris \& Pereira (1998). Em relação às restingas de todo o Estado do Espírito Santo, o presente trabalho acrescenta 28 espécies à lista de plantas vasculares apresentada por Pereira \& Araujo (2000).

A importância da família Myrtaceae na costa Atlântica brasileira é citada por Peixoto \& Gentry (1990) e Fabris \& César (1996). Esta também apresenta maior riqueza em outros estudos sobre vegetação na restinga e Mata Atlântica (Mori et al. 1981; Silva \& Leitão Filho 1982; Mantovani 1992; Bastos 1996; Thomaz \& Monteiro 1997; Pereira et al. 1998; Simonelli 1998; Assumpção \& Nascimento 2000; Pereira et al. 2000, dentre outros).

Orchidaceae e Bromeliaceae na floresta de Setiba contribuem com muitas espécies, principalmente epífitas, fato também verificado nos estudos de De Grande \& Lopes (1981), Silva \& Oliveira (1989), Mantovani (1992), Rossoni \& Baptista (1994/1995), Pereira \& Zambom (1998), Waechter (1998), Assumpção \& Nascimento (2000) e Pereira \& Araujo (2000), onde estas comumente ocorrem entre as principais famílias em número de espécies. Outras famílias com espécies de hábito epifítico na floresta de restinga do PEPCV são Cactaceae, Piperaceae e Araceae, esta última apresentando grande riqueza nas restingas ao norte do Estado do Espírito Santo (Pereira \& Gomes 1994; Pereira et al. 1998).

As famílias com maior riqueza neste estudo (Myrtaceae, Orchidaceae, Bromeliaceae e Rubiaceae) destacam-se sob este aspecto nos trabalhos acima mencionados ao longo da costa brasileira, podendo ser caracterizadas como as principais famílias das restingas brasileiras, enquanto Sapotaceae está presente dentre as de maior riqueza apenas em Setiba, constatado neste estudo e por Fabris \& César (1996).

A presença de Sapotaceae dentre as mais ricas em espécies na restinga de Setiba poderia ser explicada pela sua proximidade com áreas de Mata Atlântica, onde esta família também apresenta elevada riqueza (Peixoto \& Gentry 1990; Thomaz \& Monteiro 1997; Simonelli 1998); no entanto, em outras restingas no Estado do Espírito Santo o número de espécies de Sapotaceae não é expressivo (Pereira \& Gomes 1994; Pereira \& Zambom 1998; Pereira \& Assis 2000), demonstrando uma peculiaridade desta floresta de restinga ao sul do Estado. No estudo de Fabris \& César (1996), considerando apenas espécies arbóreas no Parque de Setiba, Sapotaceae apresentou-se como a segunda família de maior riqueza. Esta elevada riqueza de Sapotaceae na restinga de Setiba pode estar relacionada à ligação pretérita entre a flora do Estado do Espírito Santo e a da região amazônica, reconhecida por diversos autores (Prance 1979; Silva \& Shepherd 1986; Rizzini 1997), onde a família é uma das mais importantes (Leitão Filho 1987).

Lauraceae, uma das famílias com maior riqueza na floresta de restinga estudada, não figura dentre as principais famílias neste aspecto em outras áreas do litoral brasileiro. Apenas nos trabalhos de Mantovani (1992), em São Paulo, e nos de Fabris \& César (1996) e Pereira \& Assis (2000), no Espírito Santo, esta encontra-se dentre as cinco famílias mais ricas, apesar de sua importância na Mata Atlântica do próprio Estado (Peixoto \& Gentry 1990; Thomaz \& Monteiro 1997; Simonelli 1998) e em outras localidades brasileiras (Silva \& Leitão Filho 1982; Lima et al. 1997; Mantovani 1998).

Embora Leguminosae represente importante família em diversos estudos realizados na costa brasileira (Silva \& Oliveira 1989; Oliveira-Filho \& Carvalho 1993; Pereira \& Araujo 2000; Pereira \& Assis 2000), no PEPCV não ocorre esta relevância, ocupando apenas a $7^{\text {a }}$ colocação em número de espécies, juntamente com Araceae, Moraceae e Sapindaceae. Esta baixa riqueza de Leguminosae foi também encontrada por Fabris \& César (1996) em outro trecho de floresta do Parque.

$\mathrm{Na}$ análise da distribuição geográfica das espécies inventariadas em aproximadamente 4ha de floresta de restinga no PEPCV (Tab. 1) foram desconsideradas 14 espécies por apresentarem determinação incompleta: com "cf." ou "aff.", cinco táxons; em gênero, oito táxons; em família, um táxon. O padrão de distribuição geográfica mais importante em termos percentuais foi aquele onde as espécies são restritas à costa Atlântica, representando $52 \%$ do total de espécies consideradas (Fig. 1; Tab. 1), valor próximo aos $50 \%$ encontrado por Araujo (2000) analisando cerca de 500 espécies das restingas do Estado do Rio de Janeiro, e aos 53,3\% mencionados por Mori et al. (1981) em sua compilação 
194 Assis, Thomaz \& Pereira: Florística de um trecho de floresta de restinga no município de Guarapari, Espírito Santo...

Tabela 1. Lista florística de uma floresta de restinga do Parque Estadual Paulo César Vinha, Setiba, município de Guarapari (ES). (N. coletor: $\mathrm{A}=$ André M. Assis; Padrões de distribuição geográfica: $\mathrm{CA}=$ ocorrência restrita à costa Atlântica; $\mathrm{CC}=$ ocorrência na costa Atlântica e na região central do Brasil; AMP = ampla distribuição pelo território brasileiro; DIS = disjunção entre a costa Atlântica e a região Amazônica; RES $=$ restrita ao Estado do Espírito Santo).

\begin{tabular}{|c|c|c|c|c|c|}
\hline Famílias/Espécies & N. coletor & Padrão & Famílias/Espécies & N. coletor & Padrão \\
\hline AMARYLLIDACEAE & & & Crataeva tapia $\mathrm{L}$. & $A-265$ & AMP \\
\hline Amaryllis reticulata L'Her & $A-448$ & $\mathrm{CA}$ & CECROPIACEAE & & \\
\hline ANACARDIACEAE & & & Coussapoa microcarpa (Schott) Rizzini & $\mathrm{A}-832$ & $\mathrm{CC}$ \\
\hline Schinus terebinthifolius Raddi & $A-885$ & $\mathrm{CC}$ & CELASTRACEAE & & \\
\hline ANNONACEAE & & & Maytenus obtusifolia Mart. & $\mathrm{A}-874$ & $\mathrm{CC}$ \\
\hline Annona acutiflora Mart. & $A-698$ & $\mathrm{CA}$ & CLUSIACEAE & & \\
\hline Oxandra nitida R.E.Fr. & $A-721$ & $\mathrm{CA}$ & Clusia hilariana Schldtl. & A - 729 & $\mathrm{CA}$ \\
\hline Xylopia laevigata (Mart.) R.E.Fr. & $A-717$ & $\mathrm{CC}$ & C. spiritu-sanctensis G. Maris \& Weinberg & $A-527$ & $\mathrm{CA}$ \\
\hline APOCYNACEAE & & & Garcinia brasiliensis Mart. & $\mathrm{A}-751$ & AMP \\
\hline Aspidosperma parvifolium A. DC. & $A-615$ & AMP & Kielmeyera albopunctata Saadi & $A-392$ & CA \\
\hline Rauvolfia grandiflora Mart. ex. A. DC. & $A-351$ & CA & COMBRETACEAE & & \\
\hline R. mattfeldiana Markgr. & $A-655$ & $\mathrm{CC}$ & Buchenavia capitata (Vahl.) Eichler & $A-770$ & $\mathrm{CC}$ \\
\hline ARACEAE & & & COMMELINACEAE & & \\
\hline Anthurium aff. olfersianum Kunth & $A-399$ & - & Dichorisandra thyrsiflora Mikan & $A-447$ & CA \\
\hline A. pentaphyllum (Aubl.) G. Don & $A-763$ & DIS & COSTACEAE & & \\
\hline A. solitarium (Vell.) Schott & $A-404$ & CA & Costus arabicus L. & $\mathrm{A}-402$ & AMP \\
\hline Monstera adansonii Schott & $A-728$ & AMP & EBENACEAE & & \\
\hline ARECACEAE & & & Diospyros cf. janeirensis Sandwith & $A-827$ & - \\
\hline Attalea humilis Mart. & $A-569$ & $\mathrm{CC}$ & ERYTHROXYLACEAE & & \\
\hline Bactris vulgaris Barb. Rodr. & $A-869$ & $\mathrm{CA}$ & Erythroxylum oxypetalum O.E. Schulz & $A-715$ & $\mathrm{CC}$ \\
\hline Desmoncus ortacanthus Mart. & $A-818$ & AMP & E. subssessile (Mart.) O.E. Schulz & $A-497$ & $\mathrm{CA}$ \\
\hline BIGNONIACEAE & & & Erythroxylum sp. & $A-566$ & - \\
\hline Jacaranda puberula Cham. & $A-604$ & $\mathrm{CC}$ & EUPHORBIACEAE & & \\
\hline BOMBACACEAE & & & Pera glabrata (Schott) Baill. & $A-623$ & AMP \\
\hline Eriotheca pentaphylla (Vell. \& & $A-356$ & $\mathrm{CA}$ & IRIDACEAE & & \\
\hline Schum.) A. Robyns & & & Neomarica northiana (Schneev.) Sprague & $A-478$ & $\mathrm{CA}$ \\
\hline Pseudobombax grandiflorum (Cav.) & $A-537$ & AMP & LAURACEAE & & \\
\hline A. Robyns & & & Ocotea aff. bicolor Vatt-Gil & $A-384$ & - \\
\hline BROMELIACEAE & & & $O$. aff. diospyrifolia (Meisn.) Mez & $A-878$ & - \\
\hline Aechmea bromeliifolia (Rudge) Baker & $A-836$ & AMP & O. glauca (Ness) Mez & $A-484$ & $\mathrm{CA}$ \\
\hline A. lingulata (L.) Baker & $A-506$ & AMP & O. lobbii (Meisn.) Rohwer & $A-618$ & $\mathrm{CA}$ \\
\hline A. nudicaulis (L.) Griseb. & $A-400$ & CA & Ocotea sp. 1 & $A-629$ & - \\
\hline A. ramosa Mart. ex Schultz & $A-504$ & $\mathrm{CA}$ & Ocotea sp. 2 & $A-876$ & - \\
\hline Billbergia euphemiae E. Morren & A - 389 & $\mathrm{CA}$ & Rhodostemonodaphne capixabensis & $A-815$ & RES \\
\hline B. tweedieana Baker & $A-570$ & $\mathrm{CA}$ & Baitello \& Coe-Teix. & & \\
\hline Bromelia antiacantha Bertol. & $A-872$ & $\mathrm{CC}$ & LEGUMINOSAE & & \\
\hline Neoregelia macrosepala L. B. Sm. & $A-346$ & RES & CAESALPINIOIDEAE & & \\
\hline Portea petropolitana (Wawra) Mez & $A-505$ & $\mathrm{CA}$ & Hymenaea rubriflora Ducke & $A-719$ & $\mathrm{CA}$ \\
\hline Pseudananas sagenarius (Arruda) & $A-733$ & $\mathrm{CC}$ & MIMOSOIDEAE & & \\
\hline Camargo & & & Inga capitata Desv. & $A-871$ & DIS \\
\hline Tillandsia gardneri Lindl. & $A-831$ & $\mathrm{CC}$ & FABOIDEAE & & \\
\hline T. stricta Sol. & $A-880$ & $\mathrm{CC}$ & Andira nitida Mart. ex Benth. & $A-835$ & $\mathrm{CA}$ \\
\hline T. usneoides (L.) L. & $A-503$ & AMP & Exostyles venusta Schott & $A-614$ & $\mathrm{CA}$ \\
\hline $\begin{array}{l}\text { Vriesea procera (Mart. ex Schult. F.) } \\
\text { Wittm. }\end{array}$ & $A-780$ & $\mathrm{CA}$ & $\begin{array}{l}\text { Zollernia glabra (Spreng.) Yakovlev } \\
\text { LOGANIACEAE }\end{array}$ & $A-750$ & $\mathrm{CA}$ \\
\hline BURSERACEAE & & & Strychnos sp. & $A-245$ & - \\
\hline Protium heptaphyllum (Aubl.) Marchand & $A-769$ & AMP & MALPIGHIACEAE & & \\
\hline CACTACEAE & & & Byrsonima bahiana W.R. Anderson & $A-755$ & $\mathrm{CA}$ \\
\hline Epiphyllum phyllanthus (L.) Haw. & $A-248$ & AMP & Peixotoa hispidula A. Juss. & $A-534$ & $\mathrm{CA}$ \\
\hline Opuntia brasiliensis (Willd.) Haw. & $A-444$ & $\mathrm{CA}$ & MALVACEAE & & \\
\hline Rhipsalis floccosa Saslm-Dyck ex. Pfeiff. & $A-731$ & $\mathrm{CC}$ & Abutilon inaequale (Link \& Otto) & $A-560$ & $\mathrm{CA}$ \\
\hline CAPPARACEAE & & & K. Schumann & & \\
\hline Capparis baduca $\mathrm{L}$. & $A-713$ & $\mathrm{CA}$ & Pavonia alnifolia A. St.-Hil. & $A-533$ & CA \\
\hline C. flexuosa (L.) L. & $A-826$ & AMP & & & \\
\hline
\end{tabular}


Tabela 1 (continuação)

\begin{tabular}{|c|c|c|}
\hline Famílias/Espécies & N. coletor & Padrão \\
\hline \multicolumn{3}{|l|}{ MARANTACEAE } \\
\hline Maranta divaricata Roscae & $A-486$ & $\mathrm{CA}$ \\
\hline \multicolumn{3}{|l|}{ MARCGRAVIACEAE } \\
\hline Norantea brasiliensis Choisy & $\mathrm{A}-781$ & $\mathrm{CC}$ \\
\hline \multicolumn{3}{|l|}{ MELASTOMATACEAE } \\
\hline Miconia rigidiuscula Cogn. & $A-501$ & $\mathrm{CC}$ \\
\hline Mouriri arborea Gardner & $A-789$ & $\mathrm{CC}$ \\
\hline \multicolumn{3}{|l|}{ MELIACEAE } \\
\hline Guarea macrophylla Vahl & $A-526$ & AMP \\
\hline Trichilia pallens C. DC. & $A-748$ & $\mathrm{CA}$ \\
\hline T. pseudostipularis (A. Juss.) C. DC. & $A-712$ & $\mathrm{CA}$ \\
\hline \multicolumn{3}{|l|}{ MONIMIACEAE } \\
\hline Mollinedia glabra (Spreng.) Perkins & $A-754$ & $\mathrm{CA}$ \\
\hline \multicolumn{3}{|l|}{ MORACEAE } \\
\hline $\begin{array}{l}\text { Chlorophora tinctoria (L.) Benth. \& } \\
\text { Hook. }\end{array}$ & A -877 & $\mathrm{CC}$ \\
\hline Ficus clusiifolia Schott & $A-875$ & AMP \\
\hline F. cyclophylla (Miq.) Miq. & A -879 & AMP \\
\hline F. hirsuta Schott & A -873 & $\mathrm{CC}$ \\
\hline Sorocea hilarii Gaudich. & $A-259$ & $\mathrm{CA}$ \\
\hline \multicolumn{3}{|l|}{ MYRSINACEAE } \\
\hline Myrsine guianensis (Aubl.) O. Kuntze & $A-829$ & AMP \\
\hline M. parvifolia (A. DC.) Mez & $A-482$ & $\mathrm{CC}$ \\
\hline \multicolumn{3}{|l|}{ MYRTACEAE } \\
\hline Campomanesia guaviroba (DC.) Kiaersk. & $A-787$ & $\mathrm{CC}$ \\
\hline C. guazumifolia (Cambess.) O. Berg & $A-385$ & $\mathrm{CC}$ \\
\hline Eugenia bahiensis O. Berg & A -776 & $\mathrm{CA}$ \\
\hline E. aff. catharinae O. Berg & $A-493$ & - \\
\hline E. cyclophylla O. Berg & $A-822$ & $\mathrm{CA}$ \\
\hline E. cymatodes $\mathrm{O}$. Berg & $A-603$ & $\mathrm{CA}$ \\
\hline E. excelsa O. Berg & $A-724$ & $\mathrm{CA}$ \\
\hline E. ilhensis O. Berg & $A-755$ & $\mathrm{CA}$ \\
\hline E. macrantha O. Berg & $A-523$ & $\mathrm{CA}$ \\
\hline E. monosperma Vell. & $A-720$ & CA \\
\hline E. punicifolia (H.B.K.) DC. & $A-724$ & AMP \\
\hline E. rostrata $\mathrm{O}$. Berg & $A-778$ & $\mathrm{CA}$ \\
\hline E. speciosa Cambess. & $A-617$ & $\mathrm{CA}$ \\
\hline E. sulcata Spreng & $A-634$ & $\mathrm{CC}$ \\
\hline E. umbelliflora O. Berg. & $A-383$ & $\mathrm{CA}$ \\
\hline Eugenia sp. & $A-830$ & - \\
\hline Gomidesia martiana O. Berg & $A-701$ & $\mathrm{CA}$ \\
\hline Marlierea grandifolia $\mathrm{O}$. Berg & $A-562$ & $\mathrm{CA}$ \\
\hline Myrcia acuminatissima $\mathrm{O}$. Berg. & $A-767$ & $\mathrm{CA}$ \\
\hline M. bergiana $\mathrm{O}$. Berg & $A-702$ & CA \\
\hline M. fallax (Rich) DC. & $A-881$ & AMP \\
\hline $\begin{array}{l}\text { Myrciaria floribunda (H. West. ex Willd.) } \\
\text { O. Berg }\end{array}$ & $A-354$ & AMP \\
\hline Neomitranthes obtusa Sobral et Zambom & $A-723$ & RES \\
\hline Plinia rivularis (Cambess.) A.D. Rotman & $A-716$ & $\mathrm{CA}$ \\
\hline Psidium macahense O. Berg & $A-479$ & $\mathrm{CA}$ \\
\hline \multicolumn{3}{|l|}{ NYCTAGINACEAE } \\
\hline Guapira hirsuta (Choisy) Lundell & $A-564$ & $\mathrm{CA}$ \\
\hline G. laxiflora (Choisy) Lundell & A - 349 & $\mathrm{CA}$ \\
\hline G. obtusata (Jacq.) Litle & $A-882$ & $\mathrm{CC}$ \\
\hline G. opposita (Vell.) Reitz & $A-621$ & $\mathrm{CC}$ \\
\hline \multicolumn{3}{|l|}{ OCHNACEAE } \\
\hline Ouratea cuspidata (A. St.-Hil.) Engl. & $A-387$ & $\mathrm{CA}$ \\
\hline Ouratea sp. & $A-559$ & - \\
\hline
\end{tabular}

\begin{tabular}{|c|c|c|}
\hline Famílias/Espécies & N. coletor & Padrão \\
\hline \multicolumn{3}{|l|}{ OLACACEAE } \\
\hline Cathedra rubricaulis Miers & $A-772$ & $\mathrm{CA}$ \\
\hline Dulacia singularis Vell. & $A-771$ & CA \\
\hline Heisteria perianthomega (Vell.) Sleumer & $A-549$ & $\mathrm{CA}$ \\
\hline \multicolumn{3}{|l|}{ OLEACEAE } \\
\hline Linociera micrantha Mart. & $A-714$ & $\mathrm{CA}$ \\
\hline \multicolumn{3}{|l|}{ ORCHIDACEAE } \\
\hline Brassavola tuberculata Hook. & $A-452$ & AMP \\
\hline $\begin{array}{l}\text { Campilocentrum micranthum } \\
\text { (Lindl.) Rolfe }\end{array}$ & $A-610$ & AMP \\
\hline Cattleya harrisoniana Batemam ex Lindl. & $A-866$ & $\mathrm{CA}$ \\
\hline Cyrtopodium gigas (Vell.) Hoehne & $A-867$ & $\mathrm{CA}$ \\
\hline $\begin{array}{l}\text { Eltroplectris calcarata (Sw.) Garay \& } \\
\text { Sweet. }\end{array}$ & $A-451$ & AMP \\
\hline Epidendrum latilabrum Lindl. & $A-556$ & $\mathrm{CA}$ \\
\hline Habenaria leptoceras Hook & A -529 & CA \\
\hline Malaxis parthonii Morren & $A-494$ & CA \\
\hline Notylia pubescens Lindl. & $A-385$ & $\mathrm{CA}$ \\
\hline Oeceoclades maculata (Lindl.) Lindl. & $A-450$ & AMP \\
\hline Oncidium ciliatum Lindl. & $A-572$ & $\mathrm{CA}$ \\
\hline $\begin{array}{l}\text { Pleurothallis ramphastorhyncha (Barb. } \\
\text { Rodr.) Cogn. }\end{array}$ & A -394 & $\mathrm{CA}$ \\
\hline P. saundersiana $\mathrm{Rchb}$. F. & $A-525$ & $\mathrm{CA}$ \\
\hline \multicolumn{3}{|l|}{ PIPERACEAE } \\
\hline Peperomia pereskiafolia (Jacq.) Humb. & $A-507$ & $\mathrm{CC}$ \\
\hline P. rupestris H.B.K. & $A-496$ & DIS \\
\hline Piper amalago var. medium (Jacq.) Yunck. & $A-741$ & $\mathrm{CC}$ \\
\hline P. anonaefolium Kunth & $\mathrm{A}-348$ & DIS \\
\hline \multicolumn{3}{|l|}{ POLYGONACEAE } \\
\hline Coccoloba alnifolia Casar. & $A-386$ & $\mathrm{CA}$ \\
\hline \multicolumn{3}{|l|}{ RUBIACEAE } \\
\hline Amaioua guianensis A. DC. & A -883 & AMP \\
\hline Chioccoca alba (L.) Hitch. & $A-498$ & AMP \\
\hline Geophila repens (L.) Johnst. & $A-773$ & AMP \\
\hline $\begin{array}{l}\text { Posoqueria latifolia (Rudge) Roem. \& } \\
\text { Schult. }\end{array}$ & $A-557$ & AMP \\
\hline Psychotria bahiensis DC. & $A-382$ & DIS \\
\hline Randia armata DC. & $A-530$ & AMP \\
\hline Rudgea reticulata Benth. & $A-624$ & $\mathrm{CA}$ \\
\hline \multicolumn{3}{|l|}{ RUTACEAE } \\
\hline $\begin{array}{l}\text { Conchocarpus longifolius (St. Hil.) } \\
\text { Kalunki \& Pirani }\end{array}$ & $A-727$ & $\mathrm{CA}$ \\
\hline Rauia nodosa (Engl.) Kallunki & $A-256$ & $\mathrm{CA}$ \\
\hline \multicolumn{3}{|c|}{ SAPINDACEAE } \\
\hline Allophylus puberulus (A. St.-Hil.) Radlk. & $A-630$ & $\mathrm{CC}$ \\
\hline Cupania emarginata Cambess. & $A-718$ & $\mathrm{CA}$ \\
\hline Matayba guianensis Aubl. & $A-350$ & AMP \\
\hline Paullinia racemosa Wawra & $A-825$ & $\mathrm{CA}$ \\
\hline Serjania salzmaniana Schldl. & $A-785$ & $\mathrm{CC}$ \\
\hline \multicolumn{3}{|l|}{ SAPOTACEAE } \\
\hline Chrysophyllum januarensis Eichl. & $A-628$ & $\mathrm{CA}$ \\
\hline C. lucentifolium Cronquist & $A-528$ & DIS \\
\hline Manilkara subsericea (Mart.) Dubard & $A-602$ & $\mathrm{CA}$ \\
\hline $\begin{array}{l}\text { Micropholis venulosa (Mart. \& Eichler) } \\
\text { Pierre }\end{array}$ & A - 766 & AMP \\
\hline Pouteria caimito (Ruiz \& Pav.) Radlk. & $\mathrm{A}-884$ & DIS \\
\hline P. coelomatica Rizzini & $A-620$ & $\mathrm{CA}$ \\
\hline
\end{tabular}


Tabela 1 (continuação)

\begin{tabular}{lcc}
\hline Famílias/Espécies & N. coletor & Padrão \\
\hline $\begin{array}{l}\text { P. peduncularis (Mart. \& Eichl.) Baehni } \\
\text { Pouteria sp. }\end{array}$ & $\mathrm{A}-784$ & $\mathrm{CA}$ \\
$\quad$ Syderoxylon obtusifolium (Roem \& & $\mathrm{A}-658$ & - \\
$\quad$ Schult.) T.D. Penn. & & $\mathrm{CC}$ \\
$\quad$ Indeterminada & $\mathrm{A}-445$ & - \\
SIMAROUBACEAE & & \\
$\quad \begin{array}{l}\text { Picramnia glazioviana Engler } \\
\text { Simaba cuneata A. St.-Hil. \& Tul. }\end{array}$ & $\mathrm{A}-449$ & $\mathrm{CC}$ \\
SMILACACEAE & $\mathrm{A}-532$ & $\mathrm{CC}$ \\
$\quad$ Smilax sp. & $\mathrm{A}-520$ & - \\
\hline
\end{tabular}

sobre a flora da costa leste brasileira. Lima et al. (1997) registraram altos índices de espécies com distribuição restrita à costa Atlântica (70\%), a partir da flora vascular da Mata Atlântica de Macaé de Cima (RJ), indicando ser este um importante padrão de distribuição geográfica para espécies da região atlântica brasileira.

Parte das espécies restritas à costa Atlântica apresenta distribuição do Estado do Espírito Santo para o sul do país, como Eugenia cyclophylla, Myrcia

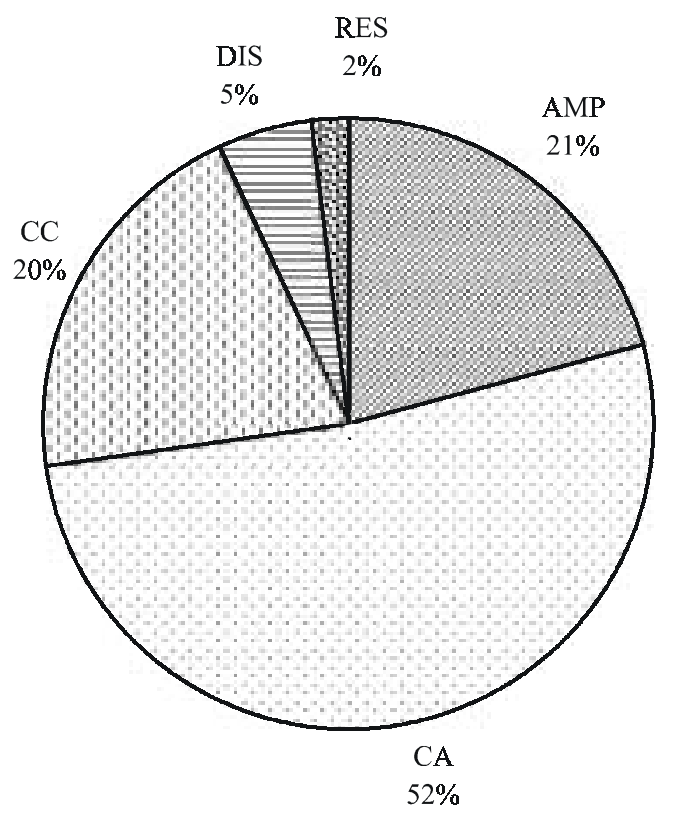

Figura 1. Padrões de distribuição geográfica das espécies ocorrentes na floresta de restinga do Parque Estadual Paulo César Vinha, Setiba, município de Guarapari - ES. (CA = ocorrência restrita à costa Atlântica; $\mathrm{CC}=$ ocorrência na costa Atlântica e na região central do Brasil; AMP = ampla distribuição pelo território brasileiro; DIS = disjunção entre a costa Atlântica e a região Amazônica; RES $=$ restrita ao Estado do Espírito Santo).

\begin{tabular}{lcc}
\hline Famílias/Espécies & N. coletor & Padrão \\
\hline $\begin{array}{l}\text { SOLANACEAE } \\
\text { Aureliana fasciculata } \text { (Vell.) Sendtn. }\end{array}$ & $\mathrm{A}-391$ & DIS \\
$\begin{array}{l}\text { Cyphomandra sycocarpa } \text { (Mart. \& } \\
\quad \text { Sendtn.) Sendtn. }\end{array}$ & $\mathrm{A}-401$ & CA \\
$\quad$ Solanum caavurana Vell. & $\mathrm{A}-268$ & $\mathrm{AMP}$ \\
$\begin{array}{l}\text { THEOPHRASTACEAE } \\
\quad \text { Clavija spinosa } \text { (Vell.) Mez }\end{array}$ & $\mathrm{A}-397$ & $\mathrm{CA}$ \\
$\begin{array}{l}\text { THYMELAEACEAE } \\
\text { Daphnopsis coriacea } \text { Taub. }\end{array}$ & $\mathrm{A}-563$ & $\mathrm{CA}$ \\
$\begin{array}{l}\text { VITACEAE } \\
\text { Cissus pulcherrima } \text { Vell. }\end{array}$ & $\mathrm{A}-442$ & $\mathrm{CA}$ \\
\hline
\end{tabular}

acuminatissima, Dulacia singularis e Pleurothalis saundersiana e algumas ocorrem do Espírito Santo para o nordeste brasileiro, como Hymenaea rubrifolia, Capparis baduca, Serjania salzmanniana e Pouteria peduncularis. Essa tendência pode estar relacionada à localização geográfica e geomorfologia costeira deste Estado, que apresenta feições diferenciadas, ora dominadas pelos tabuleiros terciários da Formação Barreiras, predominantes no nordeste brasileiro, ora pelas escarpas do Complexo Cristalino Pré-Cambriano, típico do sudeste/sul (Abreu 1943; Ruellan 1944; Suguio \& Tessler 1984). A vegetação e parte da flora das regiões nordeste e sudeste/sul são diferenciadas (Rizzini 1997), ambas contribuindo para a constituição florística do Estado do Espírito Santo, o que determina os padrões direcionais ora encontrados. Esse fato foi verificado também por Siqueira (1994) em seu estudo sobre a relação florística existente entre as diferentes áreas de Mata Atlântica estudadas no Brasil, indicando o Estado do Espírito Santo como uma área de sobreposição na distribuição geográfica entre aqueles dois blocos florísticos. Muitas das espécies com ocorrência na costa atlântica estão restritas aos Estados da Bahia, Espírito Santo e/ou Rio de Janeiro, evidenciando endemismo regional, conforme proposta de Lima et al. (1997). Esta constatação vem corroborar diversos autores (Siqueira 1994; Peixoto \& Silva 1997; Araujo et al. 1998) que incluem este trecho como um dos centros de alta diversidade e endemismo do planeta. O reconhecimento destas áreas é fundamental como base para programas de conservação ambiental, no sentido de priorizar locais de alto endemismo/diversidade: os "hotspots" (Mittermeier et al. 1999).

Outro padrão com bastante representatividade encontrado (21\%) é aquele com espécies de ampla ocorrência no território nacional, dentre estas Monstera 
adansonii, Aechmea bromelifolia, Capparis flexuosa e Myrciaria floribunda, ocorrendo nos mais variados ecossistemas brasileiros, desde o cerrado e a caatinga até a floresta equatorial Amazônica. Embora não tenha sido analisada a flora de outros países é provável que estas espécies, com ampla distribuição no território brasileiro, também ocorram em outras áreas do continente americano, de acordo com os dados obtidos por Araujo (2000), onde 95 espécies das restingas do Estado do Rio de Janeiro, correspondendo a $18 \%$ das analisadas, apresentaram este comportamento, ou seja, extrapolando os limites geográficos do Brasil, sendo 13 destas ocorrentes na floresta de restinga de Setiba.

Representando $20 \%$ do total considerado para análise, está um grupo de espécies como Jacaranda puberula, Buchenavia capitata e Campomanesia guaviroba que possui distribuição na costa Atlântica e na região central do Brasil, em ambientes como o cerrado e a caatinga, habitando, muitas vezes, florestas de galeria do cerrado, que funcionam como corredores ecológicos para a conquista de novos ambientes (Lima et al. 1997). Este tipo de distribuição geográfica representou $8,1 \%$ das espécies analisadas na Mata Atlântica de Macaé de Cima, RJ (Lima et al. 1997) e 11,8\% no estudo de Mori et al. (1981) sobre a flora do leste brasileiro.

Em menor proporção (5\%), aparecem espécies com padrão de distribuição disjunto entre a costa Atlântica e a região Amazônica, como Inga capitata, Psychotria bahiensis e Chrysophyllum lucentifolium, dentre outras. Smith (1962) mencionou possíveis rotas migratórias para as espécies com este tipo de padrão, passando pelo centro do Brasil através das matas de galerias, ou seguindo pelos Andes, penetrando no sul do país. Pereira \& Araujo (2000) sugerem haver maior correlação entre a flora das restingas do Espírito Santo em relação às do Rio de Janeiro com a da Região Amazônica, pela presença, naquele Estado, da "hiléia bahiana", conforme menções de Lima (1996, apud Peixoto \& Gentry 1990) e Rizzini (1997). Entretanto, o número de espécies disjuntas entre os dois Estados e a Amazônia, em termos percentuais, não denota grandes diferenças: $5 \%$ neste estudo e $4 \%$ no Rio de Janeiro (Araujo 2000).

As espécies ocorrentes apenas no Estado do Espírito Santo representam 2\% dentre as analisadas. Algumas ocorrem também em outros ecossistemas do Estado, como Rhodostemonodaphne capixabensis e Neoregelia macrosepala, encontradas na Floresta Atlântica (Smith \& Downs 1979; Thomaz \& Monteiro 1997; Simonelli 1998). Destaca-se dentre as restritas
Neomitranthes obtusa, espécie representada nas coleções apenas para a restinga de Setiba, nas formações florestais e na aberta de Clusia do PEPCV (Sobral \& Zambom 2002).

Segundo Pereira \& Assis (2000), as análises de distribuição geográfica das espécies de restinga são influenciadas pela escassez de trabalhos em determinados trechos da costa brasileira, principalmente na região nordeste, o que pode mascarar alguns tipos de padrões de distribuição estabelecidos.

$\mathrm{Na}$ floresta de restinga do PEPCV há ocorrência de duas espécies citadas na lista de plantas ameaçadas de extinção segundo o IBAMA: Pavonia alnifolia e Mollinedia glabra (Mello-Filho et al. 1992), além de Cattleya harrisoniana considerada por Fraga (2000) como criticamente em perigo. A presença de espécies que podem ser enquadradas em alguma das categorias de plantas ameaçadas de extinção, juntamente com outras de ocorrência restrita à restinga de Setiba (Neomitranthes obtusa), realça a importância desta unidade de conservação e justifica maior proteção deste Parque frente à ação antrópica, servindo como referência para outras áreas de restinga do Estado carentes de preservação, cuja composição florística ainda é desconhecida, como o extremo sul (e.g. Praia das Neves) e o litoral de Linhares. Fraga (2000) mencionou a necessidade de preservação deste último trecho pelo pequeno grau de pressão antrópica e a presença de espécies exclusivas de Orquidaceae.

A análise de similaridade florística entre a área estudada, com universo de 81 espécies considerando o DAP $\geq 4,8 \mathrm{~cm}$ (Assis 2001), e diferentes florestas da costa brasileira, evidenciou maior relação entre localidades próximas (Tab. 2), no próprio Estado do Espírito Santo e algumas no Rio de Janeiro, mesmo considerando outro ecossistema (Mata Atlântica). Os valores encontrados para similaridade são, em sua maioria, relativamente baixos (menores que 50\%), possivelmente relacionados às diferentes metodologias aplicadas (critério de inclusão), níveis de identificação e às próprias características dos ambientes nos estudos considerados, que englobam diferentes ecossistemas e regiões geográficas, com suas peculiaridades quanto ao clima, solo e composição florística.

No entanto, considerando as relações estabelecidas entre o presente estudo e os demais trabalhos analisados verifica-se a influência dos ambientes mais próximos na flórula local, neste caso as restingas do Rio de Janeiro e a Mata Atlântica do Estado do Espírito Santo, corroborando Cerqueira (2000) quando afirmou que cada restinga tem suas peculiaridades florísticas, devido ao 
Tabela 2. Similaridade florística entre a floresta de restinga no PEPCV (ES) e outras áreas de florestas ao longo da costa brasileira.

\begin{tabular}{|c|c|c|c|}
\hline Localidade & Ecossistema & $\mathrm{DAP}(\mathrm{cm})$ & Similaridade $(\%)$ \\
\hline Setiba, ES ${ }^{1}$ & Restinga & 4,8 & 57,9 \\
\hline Linhares, ES ${ }^{2}$ & Mata Atlântica & 5,0 & 27,1 \\
\hline São João da Barra, RJ ${ }^{3}$ & Restinga & $2,5^{*}$ & 22,0 \\
\hline Armação de Búzios, $\mathrm{RJ}^{4}$ & Restinga & 2,5 & 15,7 \\
\hline Santa Teresa, ES ${ }^{5}$ & Mata Atlântica & 10,0 & 13,5 \\
\hline Pedro Canário, $\mathrm{ES}^{6}$ & Mata Atlântica & 5,0 & 10,6 \\
\hline Cachoeira de Macacu, $\mathrm{RJ}^{7}$ & Mata Atlântica & 5,0 & 10,2 \\
\hline São Francisco de Itabapuana, RJ ${ }^{8}$ & Mata Atlântica & 10,0 & 10,1 \\
\hline Iguape, $\mathrm{SP}^{9}$ & Mata Atlântica & 5,0 & 10,1 \\
\hline Natal, RN 10 & Restinga & 4,8 & 8,7 \\
\hline Ilha do Mel, $\mathrm{PR}^{11}$ & Restinga & 4,8 & 7,6 \\
\hline Rio de Janeiro, RJ ${ }^{12}$ & Mata Atlântica & 2,5 & 7,2 \\
\hline Iguape, $\mathrm{SP}^{13}$ & Restinga & 4,8 & 6,9 \\
\hline Osório, RS ${ }^{14}$ & Restinga & 5,0 & 5,9 \\
\hline Ubatuba. SP 15 & Mata Atlântica & 10,0 & 5,5 \\
\hline Una, BA ${ }^{16}$ & Mata Atlântica & 10,0 & 4,9 \\
\hline Viamão, RS ${ }^{17}$ & Restinga & 10,0 & 4,6 \\
\hline Maracanã, PA 18 & Restinga & 2,5 & 3,2 \\
\hline
\end{tabular}

1= Fabris \& César 1996; 2= Simonelli 1998; 3= Assumpção \& Nascimento 2000; 4= Lobão \& Kurtz 2000; 5= Thomaz \& Monteiro 1997; 6= Souza et al. 1998; 7= Kurtz \& Araujo 2000; 8= Silva \& Nascimento 2001; 9= Melo et al. 2000; 10= Trindade 1991; 11= Silva et al. 1994; 12= Oliveira et al. 1995; 13= Carvalhaes 1997; 14= Dillenburg et al. 1992; 15= Silva \& Leitão Filho 1982; 16= Mori et al. 1983; 17= Waechter et al. 2000; 18= Bastos 1996. * foi utilizado o diâmetro à altura do solo (DAS).

caráter único da sua formação, ligado sobretudo a aspectos geomorfológicos. Esta ligação entre a flora das restingas e de regiões vizinhas é destacada por Rambo (1954), que mencionou a migração das espécies de ecossistemas adjacentes para a região litorânea no Rio Grande do Sul.

A restinga de Setiba apresenta características florísticas próprias, com baixa similaridade em relação a outras áreas do litoral brasileiro, com destaque para o elevado número de espécies de Sapotaceae, possivelmente oriundas da Mata Atlântica adjacente. A influência deste ecossistema nas restingas foi comprovada neste estudo com a análise da distribuição geográfica das espécies, mostrando maioria absoluta ocorrendo na Mata Atlântica (Fig. 1), reforçando a necessidade de inclusão das restingas nos programas diversos sobre a Mata Atlântica, por constituir um ecossistema associado.

A ocorrência na floresta de restinga do PEPCV de espécies endêmicas ao Espírito Santo, reforça a importância do Estado como detentor de alta diversidade e a necessidade de maior proteção desta unidade de conservação. Embora a restinga seja o ecossistema mais analisado no Estado do Espírito Santo em termos florísticos, há necessidade de ampliação da área geográfica nos estudos para fundamentar programas de conservação ambiental neste litoral.

\section{Agradecimentos}

Ao Fundo de Apoio à Ciência e Tecnologia da Prefeitura Municipal de Vitória (FACITEC), pela concessão da bolsa de estudo ao primeiro Autor; à Dra. Dorothy Sue Dunn de Araujo, pela confecção do Abstract; aos especialistas de várias famílias que identificaram grande parte do material botânico; a Luis Claudio Fabris, pela leitura crítica do manuscrito.

\section{Referências bibliográficas}

Abreu, S. F. 1943. Feições morfológicas e demográficas do litoral do Espírito Santo. Revista Brasileira de Geografia 5(2): 215-234.

Araujo, D. S. D. 1992. Vegetation types of sandy coastal plains of tropical Brazil: a first approximation. Pp. 337-347. In: U. Seeliger (ed.). Coastal plant communities of Latin America. Academic Press, New York.

Araujo, D. S. D. 2000. Análise florística e fitogeográfica das restingas do Estado do Rio de Janeiro. Tese de Doutorado. Universidade Federal do Rio de Janeiro, Rio de Janeiro.

Araujo, D. S. D. \& Henriques, R. P. B. 1984. Análise florística das restingas do Estado do Rio de Janeiro. Pp. 150-193. In: L. D. Lacerda et al. (org.). Restingas: origem, Estrutura e Processos. CEUFF, Niterói. 
Araujo. D. S. D.; Lima, H. C.; Farág, P. R. C.; Lobão, A. Q. Sá, C. C. \& Kurtz, B. C. 1998. O centro de diversidade vegetal de Cabo Frio: levantamento preliminar da flora. Pp. 147-157. In: Anais do IV Simpósio de ecossistemas brasileiros. vol. 3, ACIESP, São Paulo.

Assis, A. M. 2001. Análise florística e fitossociológica de uma formação florestal de restinga no Parque Estadual Paulo César Vinha, Setiba, município de Guarapari (ES). Dissertação de Mestrado. Universidade Federal do Espírito Santo, Vitória.

Assumpção, J. \& Nascimento, M. T. 2000. Estrutura e composição florística de quatro formações vegetais de restinga no complexo lagunar Grussaí/Iquipari, São João da Barra, RJ, Brasil. Acta Botanica Brasilica 14(3): 301-315.

Azevedo, L. G. 1962. Tipos de vegetação do Estado do Espírito Santo. Revista Brasileira de Geografia 24(1): 111-115.

Bastos, M. N. C. 1996. Caracterização das formações vegetais da restinga da Princesa, Ilha de Algodoal, Pará. Tese de Doutorado. Universidade Federal do Pará e Museu Pararense Emílio Goeldi, Belém.

Bresolin, A. 1979. Flora da restinga da Ilha de Santa Catarina. Insula 10: 1-54.

Carvalhaes, M. A. 1997. Florística e estrutura de mata sobre restinga na Juréia, Iguape, SP. Dissertação de Mestrado. Universidade de São Paulo, São Paulo.

Cerqueira, R. 2000. Biogeografia das restingas. Pp. 65-75. In: F. A. Esteves \& L. D. Lacerda (eds.). Ecologia de Restingas e Lagoas Costeiras. NUPEM/UFRJ, Macaé.

Cronquist, A. 1981. An Integrated system of classification of flowering plants. Columbia University Press, New York.

De Grande, D. A. \& Lopes, E. A. 1981. Plantas da restinga da Ilha do Cardoso (São Paulo, Brasil). Hoehnea 9: 1-22.

Dillenburg, L. R.; Waechter, J. L. \& Porto, M. L. 1992. Species composition and structure of a sandy coastal plain forest in northern Rio Grande do Sul, Brazil. Pp. 349-336. In: U. Seeliger (ed.). Coastal Plant Communities of Latin America. New York, Academic Press.

Fabris, L. C. \& Pereira, O. J. 1998. Florística da formação póspraia, na restinga de Setiba, município de Guarapari (ES). Pp.165-176. In: Anais do IV Simpósio de Ecossistemas Brasileiros. v.3, ACIESP, São Paulo.

Fabris, L. C. 1995. Composição florística e fitossociológica de uma faixa de floresta arenosa litorânea do Parque Estadual de Setiba, Município de Guarapari, ES. Dissertação de Mestrado. Universidade Estadual Paulista, Rio Claro.

Fabris, L. C. \& César, O. 1996. Estudos florísticos em uma mata litorânea no sul do estado do Espírito Santo. Boletim do Museu de Biologia Mello Leitão (Nova Série) 5: $15-46$.

Fraga, C. N. 2000. Ecologia, fitogeografia e conservação das Orchidaceae da restinga do Estado do Espírito Santo. Dissertação de Mestrado. Universidade Federal do Rio de Janeiro, Rio de Janeiro.
Kurtz, B. C. \& Araujo, D. S. D. 2000. Composição florística e estrutura do componente arbóreo de um trecho de Mata Atlântica na Estação Ecológica Estadual do Paraíso, Cachoeiras de Macacu, Rio de Janeiro, Brasil. Rodriguésia 51(78/79): 69-112.

Leitão Filho, H. F. 1987. Considerações sobre a florística de florestas tropicais e subtropicais do Brasil. Instituto de Pesquisas e Estudos Florestais 34: 41-46.

Lima, M. P. M.; Guedes-Bruni, R. R.; Sylvestre, L. S. \& Pessoa, S. V. A. 1997. Padrões de distribuição geográfica das espécies vasculares da Reserva Ecológica de Macaé de Cima. Pp. 103-123. In: H. C. Lima \& R. R. Guedes-Bruni (eds.). Serra de Macaé de Cima: Diversidade Florística e Conservação em Mata Atlântica. Jardim Botânico do Rio de Janeiro, Rio de Janeiro.

Lobão, A. Q. \& Kurtz, B. 2000. Fitossociologia de um trecho de mata de restinga na Praia Gorda, município de Armação de Búzios, RJ. Pp. 66-73. In: Anais do V Simpósio de Ecossistemas Brasileiros: conservação. v.3, ACIESP, São Paulo.

Maciel, N. C. 1990. Praias, dunas e restingas: unidades de conservação da natureza no Brasil. Pp. 326-351. In: Anais do II Simpósio de Ecossistemas da Costa Sul e Sudeste Brasileira. Estrutura, função e manejo. v.3, ACIESP, São Paulo.

Mantovani, W. 1992. A vegetação sobre a restinga de Caraguatatuba, SP. Revista do Instituto Florestal de São Paulo 4: 139-144.

Mantovani, W. 1998. Dinâmica da floresta pluvial Atlântica. Pp.1-20. In: Anais do IV Simpósio de Ecossistemas Brasileiros. v.2, ACIESP, São Paulo.

Martins, M. L. L.; Carvalho-Okano, R. M. \& Luceño, M. 1999. Cyperaceae no Parque Estadual Paulo César Vinha, Guarapari, Espírito Santo, Brasil. Acta Botanica Brasilica 13(2): 187-222.

Melo, M. M. R. F.; Oliveira, R. J.; Rossi, L.; Mamede, M. C. H. \& Cordeiro, I. 2000. Estrutura de um trecho da Floresta Atlântica de planície na Estação Ecológica Juréia-Itatins, Iguape, SP, Brasil. Hoehnea 27(3): 299-322.

Mello-Filho, L. E.; Sommer, G. V. \& Peixoto, A. L. 1992. Centuria Plantarum Brasiliensium exstintions Minitata. Sociedade Botânica do Brasil / IBAMA, Brasília.

Ministério do Meio Ambiente, 2000. Avaliação e ações prioritárias para a conservação da biodiversidade da mata atlântica e campos sulinos. MMA/SBF, Brasília.

Mittermeier, R.A.; Fonseca, G. A. B.; Rylands, A. B \& Mittermeier, C. G. 1999. Atlantic Forest. Pp. 136-144. In: P. R. Gil (coord.). Hotspots - Earth's Biological Richest and Most Endareged Terrestrial Ecorregions, CEMEX /Sierra Madre/Conservation International, Cidade do México.

Mori, S. A.; Boom, B. M. \& Prance, G. T. 1981. Distribuition patterns and conservation of the Eastern Brazilian coastal forest tree species. Brittonia 33(2): 233-245. 
Mori, S. A.; Boom, B. M.; Carvalho, A. M. \& Santos, T. S. 1983. Southern bahian moist forest. The Botanical Review 49(2): 155-232.

Mueller-Dombois, D. \& Ellenberg, H. 1974. Aims and methods of vegetation ecology. J. Wiley \& Sons, New York.

Oliveira-Filho, A. T. \& Carvalho, D. A. 1993. Florística e fisionomia da vegetação no extremo norte do litoral da Paraíba. Revista Brasileira de Botânica 16(1): 115-130.

Oliveira, R. R.; Záu, A. S; Lima, D. F.; Silva, M. B. R.; Viana, M. C.; Sodré, D. O. \& Sampaio, P. D. 1995. Significado ecológico da orientação de encostas no Maciço da Tijuca, Rio de Janeiro. Pp. 523-542. In: F. A. Esteves (ed.). Oecologia Brasiliensis: estrutura, funcionamento e manejo de ecossistemas brasileiros. v.1, UFRJ, Rio de Janeiro.

Peixoto, A. L. \& Gentry, A. 1990. Diversidade e composição florística de mata de tabuleiro na Reserva de Linhares (Espírito Santo, Brasil). Revista Brasileira de Botânica 13: $19-25$.

Peixoto, A. L. \& Silva, I. M. 1997. Tabuleiro forests of Northern Espirito Santo, South-eastern Brazil. Pp. 369-372. In: S. D. Davis et al. Centres of Plant Diversity: a guide and strategy for their conservation. v.3: The Americas. IUCN Publications Unit, Cambrige.

Pereira, O. J. 1990. Caracterização fitofisionômica da restinga de Setiba - Guarapari, ES. Pp. 207-219. In: Anais do II Simpósio de ecossistemas da costa sul e sudeste brasileira. Estrutura, função e manejo. v.3, ACIESP, São Paulo.

Pereira, O. J. \& Araujo, D. S. D. 2000. Análise florística das restingas dos Estados do Espírito Santo e Rio de Janeiro. Pp. 25-63. In: F. A. Esteves \& L. D. Lacerda (eds.). Ecologia de Restingas e Lagoas Costeiras. NUPEM/UFRJ, Macaé.

Pereira, O. J. \& Assis, A. M. 2000. Florística da restinga de Camburi. Acta Botanica Brasilica 14(1): 99-111.

Pereira, O. J.; Assis, A. M. \& Souza, R. L. D. 1998. Vegetação da restinga de Pontal do Ipiranga, Município de Linhares (ES). Pp.117-128. In: Anais do IV Simpósio de Ecossistemas Brasileiros. v.3, ACIESP, São Paulo.

Pereira, O. J.; Borgo, J. H.; Rodrigues, I. D. \& Assis, A. M. 2000. Composição florística de uma floresta de restinga no município da Serra, ES. Pp. 74-83. In: Anais do V Simpósio de Ecossistemas Brasileiros: conservação. v.3, ACIESP, São Paulo.

Pereira, O. J. \& Araujo, D. S. D. 1995. Estrutura da vegetação de entre moitas da formação aberta de Ericaceae no Parque Estadual de Setiba, ES. Pp. 245-257. In: F. A. Esteves (ed.). Oecologia Brasiliensis: estrutura, funcionamento e manejo de ecossistemas brasileiros. v.1, UFRJ, Rio de Janeiro.

Pereira, O. J. \& Gomes, J. M. L. 1994. Levantamento florístico das comunidades vegetais de restinga no Município de Conceição da Barra, ES. Pp. 67-78. In: Anais do III Simpósio de ecossistemas da costa sul e sudeste brasileira. Subsídios a um gerenciamento ambiental. v.3, ACIESP, São Paulo.
Pereira, O. J. \& Zambom, O. 1998. Composição florística da restinga de Interlagos, Vila Velha (ES). Pp. 129-139. In: Anais do IV Simpósio de ecossistemas brasileiros. v.3, ACIESP, São Paulo.

Prance, G. T. 1979. The taxonomy and phytogeography of the Chrysobalanaceae of the Atlantic coastal forest of Brazil. Revista Brasileira de Botânica 2: 19-39.

Rambo, B. 1954. História da flora do litoral riograndense. Sellowia 6: 112-172.

Rizzini, C. T. 1997. Tratado de Fitogeografia do Brasil: aspectos ecológicos, sociológicos e florísticos. Âmbito Cultural Edições LTDA, Rio de Janeiro.

Rossoni, M. G. \& Baptista, L. R. M. 1994/1995. Composição florística da mata de restinga, Balneário Rondinha, Arroio do Sal, RS, Brasil. Pesquisas 45: 115-131.

Ruellan, F. 1944. A geomorfologia do litoral espiritossantense. Boletim Geográfico 20(21): 1357-1367.

Ruschi, A. 1950. Fitogeografia do Estado do Espírito Santo I: considerações gerais sobre a distribuição da Flora no Estado do E. Santo. Boletim do Museu de Biologia Mello Leitão (Série Botânica) 1: 1-353.

Silva, A. F. \& Leitão Filho, H. F. 1982. Composição florística e estrutura de um trecho de Mata Atlântica de encosta no município de Ubatuba (São Paulo, Brasil). Revista Brasileira de Botânica 5: 43-52.

Silva, A. F. \& Shepherd, G. J. 1986. Comparações florísticas entre algumas matas brasileiras utilizando análise de agrupamento. Revista Brasileira de Botânica 5: 43-52.

Silva, G. C. \& Nascimento, M. T. 2001. Fitossociologia de um remanescente de mata sobre tabuleiros no norte do estado do Rio de Janeiro (Mata do Carvão). Revista Brasileira de Botânica 24(1): 51-62.

Silva, J. G. \& Oliveira, A. S. 1989. A vegetação de restinga no município de Maricá, RJ. Acta Botanica Brasilica 3(2): 253-272 (suplemento).

Silva, S. M.; Britez, R. M.; Souza, W. S. \& Joly, C. A. 1994. Fitossociologia do componente arbóreo da floresta de restinga da Ilha do Mel, Paranaguá, PR. Pp. 33-48. In: Anais do III simpósio de ecossistemas da costa sul e sudeste brasileira. Subsídios a um gerenciamento ambiental. v.3, ACIESP, São Paulo.

Simonelli, M. 1998. Composição florística e estrutura do estrato arbóreo de uma muçununga na Reserva Florestal de Linhares, Espírito Santo. Dissertação de Mestrado. Universidade Federal de Viçosa, Viçosa.

Siqueira, M. F. 1994. Análise florística e ordenação de espécies arbóreas da Mata Atlântica através de dados binários. Dissertação de Mestrado. Universidade Estadual de Campinas, Campinas.

Smith, L. B. 1962. Origins of the flora of Southern Brazil. Contributions from the United States National Herbarium 35(3): 215-249.

Smith, L. B. \& Downs, R. J. 1979. Bromeliaceae- Bromelioideae. Flora Neotropica Monograph 14(3): 1493-2142. 
Sobral, M. \& Zambom, O. 2002. Neomitranthes obtusa (Myrtaceae), a new species from Espirito Santo, Brazil. Novon 12(1): 112-114.

Souza, A. L.; Meira Neto, J. A. A. \& Schettino, S. 1998. Avaliação florística, fitossociológica e paramétrica de um fragmento de floresta Atlântica secundária, município de Pedro Canário, Espírito Santo. Boletim Técnico da Sociedade de Investigações Florestais n. 18: 1-117.

Suguio, K. \& Tessler, M. G. 1984. Planícies de cordões litorâneos quaternários do Brasil: Origem e nomenclatura. Pp. 15-25. In: L. D. Lacerda et al. (org.). Restingas: origem, estrutura e processos. CEUFF, Niterói.

Sugiyama, M. 1998. Estudo de florestas da restinga da Ilha do Cardoso, Cananéia, São Paulo. Boletim do Instituto de Botânica 11: 119-159.

Thomaz, L. D. \& Monteiro, R. 1997. Composição florística da Mata Atlântica de encosta da Estação Biológica de Santa Lúcia, município de Santa Teresa, ES. Boletim do Museu de Biologia Mello Leitão (Nova Série) 7: 1-48.
Trindade, A. 1991. Estudo florístico e fitossociológico do estrato arbustivo-arbóreo de um trecho de floresta arenícola costeira do Parque Estadual das Dunas Natal, RN. Dissertação de Mestrado. Universidade Federal Rural de Pernambuco, Recife.

Ule, E. 1901. Die vegetation von Cabo Frio an der Kusten von Brasilien. Botanische Jahrbiicher für Systematik 28: 511-528.

Waechter, J. L. 1985 Aspectos ecológicos da restinga do Rio Grande do Sul, Brasil. Comunicação Científica da Pontifícia Universidade Católica do Rio Grande do Sul - Série Botânica 33: 49-68.

Waechter, J. L. 1998. Epifitismo vascular em uma floresta de restinga do Brasil subtropical. Revista Ciência e Natura 43: 43-66.

Waechter, J. L; Müller, S. C.; Breier, T. B. \& Venturi, S. 2000. Estrutura do componente arbóreo de uma floresta subtropical de planície costeira interna. Pp. 92-112. In: Anais do V Simpósio de ecossistemas brasileiros: conservação. v.3, ACIESP, São Paulo. 